\title{
Posttraumatic Stress Symptoms in Young People with Cancer and their Siblings: Results from a UK sample
}

\begin{abstract}
Purpose: This study investigated levels of posttraumatic stress symptoms (PTSS) in children with cancer and their siblings from a British sample. It also examined aspects of the Ehlers and Clark ${ }^{1}$ model of posttraumatic stress disorder (PTSD) in the current population.

Methods: Sixty participants (34 children with cancer and 26 siblings) aged between 8-18 years completed measures of PTSS, maladaptive appraisals, trauma-centered identity, perceived social support and family functioning.

Results: Over a quarter of the sample scored above the clinical cut off on the Impact of Events Scale-Revised (IES-R). No differences were observed between patients and siblings with respect to levels of PTSS. Maladaptive appraisals and age were found to account for unique variance in levels of PTSS for the overall sample.
\end{abstract}

Conclusions: Rates of PTSS in the sample was relatively high. Support was found for aspects of the Ehlers and Clark ${ }^{1}$ model in explaining PTSS for the current population. 
Psychological trauma is triggered by events that are sudden, unexpected and in which the individual perceives an intense loss of personal control and safety. ${ }^{2}$ Symptoms of posttraumatic stress disorder (PTSD) as described in the Diagnostic and Statistical Manual of Mental Disorders-Fifth Edition (DSM-5) ${ }^{3}$ consist of a combination of four cluster criterions (intrusions, avoidance, negative alterations in cognitions and mood, and marked alterations in arousal and reactivity), which need to have been present for at least one month and cause significant functional impairment. ${ }^{4}$ For individuals who only partly meet the diagnostic criteria of the disorder, the terms 'sub-threshold PTSD', 'partial PTSD' or 'posttraumatic stress symptoms (PTSS) have been used. ${ }^{5}$ It is thought that cancer diagnosis and treatment can be incorporated within this definition. ${ }^{6}$

Estimates of PTSD in children with cancer vary from $4.7 \%{ }^{7}$ to $21 \% .^{8}$ Estimates of subclinical levels of PTSS have been reported as high as 78\% endorsing at least one cluster criterion. ${ }^{9}$ Furthermore, almost $70 \%$ of mothers and $60 \%$ of fathers expressed PTSS. ${ }^{10}$ The importance of understanding these symptoms is accentuated by recent studies that highlighted the functional impairment and distress associated with PTSS. Indeed, Varela et $\mathrm{al}^{11}$ found that the majority of childhood cancer survivors with partial PTSD $(86.5 \% ; n=105)$ reported experiencing functional impairment related to these sub-threshold symptoms, with the majority reporting impairment in multiple areas of functioning. Subclinical levels of PTSD can have important implications in this population group. For example, avoiding cancer related stimuli could influence decision-making regarding treatment. ${ }^{12,13}$ Furthermore, avoidance in children with life threatening illnesses significantly correlated with nonadherence to treatment regimen ${ }^{14}$ as well as the fact that patients may minimize their physical difficulties to avoid reminders of the cancer diagnosis and treatment. ${ }^{15,16}$ The presence of PTSS has also been found to influence patients with general medical illnesses' healthcare 
utilization and medical outcome. ${ }^{17}$ It has been noted that the cancer experience has the potential to bestow a series of traumatic events over time. ${ }^{18}$ This can include diagnosis (including disease progression) and treatment/treatment complications (including invasive procedures). Furthermore, other potentially traumatic events can also occur during the course of treatment, including the death of other children known to the family, emergency surgery or admission to intensive care units, as well as the threat of relapse. ${ }^{7}$ This can have an impact on the young person with cancer, as well as their families. Indeed, Kazak and colleagues have described symptoms of PTSD as 'one of the most important psychological consequences of childhood cancer' (p. 493). ${ }^{7}$

The Diagnostic and Statistical Manual of Mental Disorders, $4^{\text {th }}$ edition (DSM-IV; APA 1994) broadened its taxonomy of PTSD to include being 'diagnosed with a life threatening illness' or 'learning that one's child' ${ }^{19}$ (p. 426) has the aforementioned illness, as a qualifying stressful event. ${ }^{20}$ Furthermore, DSM-5 continues to posit that medical incidents involving 'sudden, catastrophic events' qualify as traumatic stressors ${ }^{3}$ (p. 274).

Guidelines from the National Institute for Health and Clinical Excellence ${ }^{21}$ aimed at improving outcomes in childhood cancer highlight the importance of psychological services in cancer treatment. It is therefore crucial to investigate rates of PTSS in this population and to highlight factors that may contribute to this in order to provide effective psychological treatments for this population group.

The majority of previous research examining PTSD associated with childhood cancer has focused on patients and their parents (particularly mothers). However, a recent systematic review examining psychological functioning in siblings of children with cancer concluded that a PTSD framework may provide a useful way of conceptualizing sibling distress. ${ }^{22}$ Research in this area remains scant and the review identified only five studies examining these symptoms in siblings of children with cancer. These studies reported that between $29 \%$ 
and $38 \%$ of siblings reported moderate to severe PTSS, a rate greater than that found in a comparison sample of healthy children. ${ }^{23}$ Study limitations identified included failure to detail the time since diagnosis and incorporating select samples (e.g. siblings attending a summer camp). Furthermore, the studies did not provide information on which factors were associated with increased risk of PTSS, thus failing to guide clinicians as to which siblings may be most likely to experience these symptoms.

Results from the Childhood Cancer Survivor Study (CCSS), a longitudinal cohort study which examined over 18,000 adult survivors of childhood cancer and their siblings, demonstrated that adult survivors of childhood cancer had more than a four-fold risk of developing PTSD compared to siblings. ${ }^{24}$ No studies were identified which compared children and adolescents with cancer and siblings on levels of PTSD.

Both Kangas and colleagues ${ }^{25}$ and Bruce $^{20}$ underscored the importance of placing cancer within a PTSD framework as informed by PTSD models. The cognitive model of PTSD $^{1}$ reports that pathological responses to trauma arise when individuals process the traumatic information in a method that produces a sense of current threat. This is maintained by negative appraisals of the trauma and its sequeale, as well as the nature of the trauma memory. Negative appraisals can be focused on the traumatic event itself, of one's own actions, of others people's reactions or of life prospects. The importance of maladaptive behavioral and cognitive strategies in maintaining the disorder is also highlighted. ${ }^{26}$

Evidence is accumulating regarding the relevance of cognitive models to explain the development and maintenance of trauma symptoms within adult and child populations. ${ }^{27-29}$ Certain aspects of these models have been found to be particularly useful within child populations. These include the role of maladaptive appraisals and trauma-centered identity in predicting PTSD. ${ }^{30}$ Furthermore, the inclusion of parental and environmental factors in order address distinct developmental issues has been highlighted by a number of authors. ${ }^{31-33}$ 
These environmental factors include the child's perceived social support as well as understanding the family's functioning. ${ }^{34}$ A number of reviews have highlighted that these factors can moderate the impact of the traumatic event, the impact of trauma reminders as well as the influence of ongoing stressors. ${ }^{30,35}$ It has been suggested that future research should conceptualize psychological distress in response to cancer using models such as the cognitive appraisal model of $\mathrm{PTSD}^{1}$ to guide research and facilitate specific hypothesestesting. ${ }^{16}$

The aims of this study were two-fold. Firstly, the study aimed to examine whether there were any differences between patients and siblings in levels of PTSS, as well as to investigate the levels of PTSS for both groups in this study. This has implications for service provision in pediatric psychology services in the UK. Secondly, the current research aimed to explore the impact of maladaptive cognitive appraisals and trauma-centered identity on PTSS, as indicated by the cognitive model of PTSD ${ }^{1}$ for both children with cancer and their siblings. Specifically, we hypothesized that cognitive (i.e. maladaptive appraisals and trauma centered identity) and environmental variables (i.e. family functioning and perceived social support) would explain variance in levels of PTSS for the current sample.

\section{Methods}

\section{Participants}

Participants included 60 children aged 8-18 years $(M=12.38, S D=2.85)$. This included 34 patients and 26 siblings. One parent per family was also included to complete an assessment of family functioning. Inclusion criteria were: both patients and siblings aged 8-18 years at time of recruitment, patients (and siblings of patients) at least three months post diagnosis and no more than five years post treatment. Exclusion criteria were: severe cognitive impairment, patient deceased or receiving palliative care (or sibling of child receiving 
palliative care) and/or lack of fluency in English. Of the 34 patients who completed the study, $11(32 \%)$ were currently receiving treatment. Of the 52 patients who were approached by staff during outpatient appointments, 31 families consented to be contacted (60\%). Five families declined participation following contact from the researcher (16\%) and 6 families contacted the researcher independently via the poster advertisement. Of the initial 52 families approached, 26 participated in the study (overall response rate of 50\%). Figure 1 provides an illustrative overview of the recruitment process. There were no significant differences in time since diagnosis or treatment duration between participants and non-participants $(p>.05)$.

Other demographic and treatment related data for non-participants were not recorded due to regulations relating to patient data protection in the hospital. Table 1 provides further information regarding the demographic and treatment variables of participants.

\section{Procedure}

The study was granted ethical approval by the Norfolk Research Ethics Committee (Ref: 13/EE/0143) in June 2013. Primarily, a member of the clinical care team reviewed the computerized register of pediatric cancer patients in order to identify families who met the initial inclusion criteria. Parents were approached during the child's usual outpatient appointment by a member of the clinical care team and were provided with an information pack explaining the study. In the case where the patient had a number of eligible siblings, the sibling closest in age to the patient was invited to take part.

If parents were interested in obtaining more information about the study, they were advised to complete a 'consent to contact' form and the author would contact them to explain the study in more detail and answer any questions they had. If parents, the patient and/or their sibling were interested in participating in the study, the author organized a time, date and location to come and complete the questionnaires. Posters that outlined inclusion/exclusion 
criteria as well as contact details for the primary researcher (AD) were also placed in the hospital waiting room. The majority of participants chose to complete the questionnaires at home $(97 \% ; \mathrm{n}=58)$, and the remainder of participants $(3 \% ; \mathrm{n}=2)$ completed the questionnaires during a hospital appointment. Children who scored above the cut off on the IES-R at the time of assessment were offered an appointment with the clinical psychologist within hospital's pediatric oncology department.

\author{
Measures \\ Child PTSS. The Impact of Events Scale-Revised (IES-R) ${ }^{36}$ was used to measure PTSS in \\ both the patient and sibling sample. This is a well-standardized, 22 item self-report \\ instrument designed to measure the three symptom clusters - intrusion, avoidance and \\ hyperarousal - associated with the DSM-IV criteria for PTSD. Participants are asked to think \\ about the part of the cancer experience which was most frightening for them and to report the \\ degree of distressed experienced for each item in the past 7 days. Items are rated from $0-4$ \\ $(0=$ not at all to $4=$ extremely $)$, relating to frequency of distress associated with intrusion \\ (e.g. "any reminder brought back feelings about it"), avoidance (e.g. "I stayed away from \\ reminders about it") and hyperarousal (e.g. "I felt watchful and on guard"). Higher scores \\ indicate increased levels of PTSS. The IES-R has been used extensively with children with \\ cancer and their families. ${ }^{7,37}$ In the current study, the data confirmed the measures high \\ internal consistency (Cronbach's $\alpha=0.86$ in the patient group and $\alpha=0.88$ in the sibling \\ group).
}

Maladaptive Appraisals. The Children's Posttraumatic Cognitions Inventory (CPTCI) ${ }^{38}$ was administered to both patients and siblings to measure maladaptive appraisals derived 
following the cancer diagnosis. The CPTCI, which was adapted from the Posttraumatic Cognitions Inventory for adults (PTCI) ${ }^{39}$ contains two subscales: permanent and disturbing change (13 items; e.g. "My life has been destroyed by the frightening event," "I will never be able to have normal feelings again") and feelings of vulnerability (12 items; e.g. "Bad things always happen," "Everyone lets me down"). Items are rated in terms of levels of agreement $(1=$ don't agree at all to $4=$ agree a lot $)$. Higher scores represent increased maladaptive appraisals. In the current study, the scale was found to have excellent internal consistency for both groups (Cronbach's $\alpha=0.90$ for the patient group and $\alpha=0.91$ for the sibling group).

Trauma-Centered Identity. The Centrality of Event 7-item Scale (CES-7) ${ }^{40}$ was administered to patients and siblings to measure how central the traumatic event(s) was to their identity and life story (e.g. "the frightening event is part of who I am now" and "if I were to tell a story about my life, the frightening event would be one of the most important parts"). Items are rated on a 4-point scale indicating levels of agreement with each statement. An adapted version of the scale for use with children who had experienced a traumatic event was employed (CCES). In the current study, the data confirmed the measures high internal consistency (Cronbach's $\alpha=0.84$ in the patient group and $\alpha=0.85$ in the sibling group).

Family Functioning. Family functioning was measured using the 12-item Global Functioning Scale (GFS), which forms part of the Family Assessment Device (FAD) ${ }^{41}$ This was completed by the patient's or sibling's primary caregiver. This scale is designed to assess problematic family functioning ${ }^{41}$ (e.g. "We avoid discussing our fears and concerns," "In times of crisis, we can turn to each other for support"). The scale requires caregivers to rate the degree to which each statement describes their family, with responses on a 4-point Likert type scale, $(1=$ "strongly agree" and $4=$ "strongly disagree"). Scores are reversed for certain 
items and lower scores reflect better functioning. The FAD emerged as one of the most appropriate tools for assessment of family functioning in pediatric pain populations. ${ }^{42}$ In the current study, the internal consistency of the FAD was established as $\alpha=0.87$.

Perceived Social Support. Perceived social support was assessed utilizing the Multidimensional Scale of Perceived Social Support (MSPSS ${ }^{43}$ for both patients and siblings. The MSPSS is a 12-item questionnaire used to measure how one perceives their social support system as well as the rating of sources of social support. Items are rated on a 7point scale $(1=$ very strongly disagree and $7=$ very strongly agree $)$. Items relate to the perceived social support derived from various individuals in the participant's social system, including family (e.g. "I can talk about my problems with my family"), friends (e.g. "I can count on my friends when things go wrong") and significant others (e.g. "There is a special person who is around when I am in need"). Higher scores on the measure indicate higher levels of perceived social support. In the current study, the internal consistency of the MSPSS was $\alpha=0.87$ for both the patient and sibling groups.

\section{Statistical Analyses}

All analyses were conducted using SPSS statistical software, Version 22, and inspected for departures from normality. The data were screened in accordance with the recommendations outlined by Tabachnick and Fidell. ${ }^{44}$ The data were screened using both visual inspection (histogram and box plots) and statistical methods (testing of skew and kurtosis) to ensure that the data set was complete, clear of errors and to check the distribution.

Data values were missing for four of a total of 60 participants (7\%). Boxplots were produced to screen for any outliers or extreme values, in accordance with guidelines outlined by 
Pallant. ${ }^{45}$ None of the outliers identified were considered extreme points. Power calculations completed prior to data collection indicated that there was $80 \%$ power to detect correlations of $r \geq .21$ for the sample at a significance level of 0.05 . In order to control for Type 1 errors, Bonferroni adjustments (according to the method outlined by $\mathrm{Wright}^{46}$ ) were applied.

Bivariate analyses were completed to examine the relationship between PTSS and the various demographic, objective illness and psychological variables previously outlined.

Subsequently, hierarchical multi linear regression modelling was used to examine the variance in PTSS accounted for by the above variables. Variables were entered in a hierarchical fashion, with age entered in a first step, and maladaptive appraisals and traumacentered identity entered in a second step; this procedure was undertaken to consider whether psychological mechanisms improved the model over and above age. The sample was combined for bivariate and multivariate analyses in order to increase the overall statistical power to examine the relationship between psychological variables and PTSS in this population. While the authors acknowledge the important conceptual distinction between cancer patients and their sibling's experiences of cancer diagnosis and treatment, due to the study's relatively low power, the decision was made to combine the samples for statistical analyses. Furthermore, between group analyses indicated no significant difference in patients' and their siblings' demographic backgrounds, therefore it was considered to offer further justification -with accepted limitations - to the decision of combining the samples for analyses.

\section{Results}

\section{PTSD severity}

The data were not normally distributed and therefore patient and sibling groups were compared using the Mann-Whitney U test. This indicated no significant differences in levels 
of PTSS between patient $(M d=29)$ and sibling $(M d=33)$ samples, $U=389, p=.43, r=.10$. A Wilcoxon signed-rank test was conducted to evaluate differences in levels of PTSS between patient and sibling pairs (i.e. pairs from the same family; $n=44$ ). There was no significant difference in levels of PTSS between patient $(M d=23)$ and sibling $(M d=23)$ pairs $(Z=-1.218, p=.223)$.

A total of $28 \%(n=17)$ of the overall sample; $31 \%$ of the sibling group $(n=8)$ and $27 \%$ of the patient group $(n=9)$ scored above the cut off $(\geq 33)$ on the IES-R $\left(\chi^{2}=.134\right.$, $\mathrm{p}=.714$ ). The primary stressor identified by participants is outlined in Table 2 ; in the majority of instances the primary stressor was the actual diagnosis.

\section{Bivariate Analyses}

Significant positive correlations were found between levels of PTSS in the total sample and age, maladaptive appraisals (both 'permanent and disturbing change' and 'fragile person in a scary world' appraisals) and trauma centered identity $(p<0.05)$. No other demographic, illness or psychosocial factor correlated significantly with levels of PTSS (see Table 3) in the total sample, but perceived social support was significantly and positively correlated with PTSS in the sibling group. There was no significant difference between patients and siblings with respect to gender.

\section{Multivariate Analyses}

Results from the multivariate analyses are displayed in Table 4 . The entry of age in the first step did significantly improve the model, accounting for $14.7 \%$ of variance; the addition of the CPTIC and CES also further improved the model (for a total of $36.8 \%$ of variance).

However, the only variable to account for unique variance in the final model was maladaptive appraisals (beta $=.38)$. 
This model was repeated but with child's status (i.e. 'patient' or 'sibling') also entered in the first step. This model was undertaken to ensure that this variable did not alter the pattern of results. In this model, the first step account for $15.6 \%$ of variance, but only age and not child status accounted for unique variance at this point. The second step also further improved the model (for a total of $38.4 \%$ of variance). As before, only maladaptive appraisals (beta $=.37$ ) accounted for unique variance in the final model.

\section{Discussion}

The current study aimed to examine any potential differences in the levels of PTSS in a British sample of children with cancer and their siblings. It also aimed to examine whether cognitive aspects of the Ehlers-Clark ${ }^{1}$ cognitive model of PTSD could be considered applicable in this population.

Although there were no significant differences in the levels of PTSS between children with cancer and their siblings, the percentage of participants meeting cut-off for a potential diagnosis of PTSD was substantial, with $27 \%$ of patients and $31 \%$ of siblings indicating potential PTSD 'caseness' ${ }^{47}$ The individual symptoms assessed, albeit not achieving diagnostic status, may have important implications. For instance, avoidance in children with life threatening illnesses significantly correlated with non-adherence to treatment regimen. ${ }^{14}$

Second, the relatively high rate of PTSS in siblings highlights the importance of ensuring service provision for those individuals who may require psychological input. Variable intervention provision in the UK may shed light on the higher rates of PTSS demonstrated for this population compared to samples derived from the US. ${ }^{21,} 23,48$

Research with adult survivors of childhood cancer suggests that the cancer experience and late effects associated with treatment results in these individuals having a four-fold 
greater risk of developing PTSS compared to their siblings. ${ }^{24}$ The authors were unable to identify any other studies that directly compared levels of PTSS in children with cancer and their siblings. The comparable rates of PTSS in children with cancer and siblings may be due to the shared environmental and familial factors previously mentioned. Although not assessed in the current study, previous research has indicated that parents of children with cancer have a heightened risk of developing PTSS $^{7}$, which may have an impact on levels of PTSS expressed by both patients and siblings. These shared environmental and familial factors may be less prevalent for adult survivors and their siblings. Indeed, a qualitative review ${ }^{49}$ highlighted three main areas of stressors influencing young siblings' wellbeing. These included; (i) tremendous changes in their lives, particularly with regards to family relationships and routines, (ii) facing the constant unpredictability of the disease and (iii) feeling isolated and on the periphery of family life.

Participants within the current sample endorsed a range of maladaptive appraisals. These appraisals are thought to maintain PTSD by generating negative emotions and encouraging individuals to engage in dysfunctional coping strategies. ${ }^{1}$ Given the robust correlations between maladaptive appraisals and PTSS in the current study, as well as the excellent internal consistency of the CPTCI measure in the sample, it may be that this measure can prove clinically useful in attempts to identify the nature of young people's maladaptive appraisals about cancer.

Within their proposed cognitive model of maintenance, Ehlers \& Clark $^{1}$ also aim to explain a 'frozen in time' phenomenon whereby individuals with chronic symptoms of PTSD describe feeling locked into their past. It may be that this experience relates to cognitions regarding 'permanent and disturbing change' explored within this study. Indeed, it is proposed that patients may sense they have permanently changed (as described in the current study). This may cause young people to find it difficult to return to activities that they 
considered important before the traumatic event, which contributes to the sense that their life hasn't moved on. This is in line with participant's agreement on questions such as 'My life has been destroyed by the frightening event'. This has important implications for individuals diagnosed with cancer. Indeed, previous research has indicated that perceived social competency and self-esteem are lower in children with cancer who are off treatment than children who are on treatment. ${ }^{50,51}$ Impairments in social competence have also been reported for siblings of children with cancer. ${ }^{52}$ This may indicate that children find it more difficult to return to activities that they previously enjoyed, even once they have completed treatment. ${ }^{53}$ This may prevent alterations in maladaptive appraisals of the traumatic event.

The fact that illness-related factors were not associated with levels of PTSS supports findings from previous studies. ${ }^{7}$ 16, 54 Importantly, time since diagnosis, treatment duration and treatment status (on/off treatment) were not found to be associated with PTSS, suggesting that these symptoms are more than just stress reactions in response to cancer diagnosis. This finding indicates that early identification of cancer patients who are likely to require mental health assistance should focus on psychological as well as medical variables. This supports previous research within the child and adult psycho-oncology literature. ${ }^{55}$

Findings from the current study implicating the role of cognitive variables, supports the potential application of the Surviving Cancer Competently Intervention Program - Newly Diagnosed (SSCIP - ND) to patients and siblings. The SSCIP-ND represents a three-session manualized treatment program devised for parents of children newly diagnosed with cancer. ${ }^{56}$ This program integrates cognitive-behavioral and family therapy principles by identifying and altering the parent beliefs that may facilitate or hinder adaptive functioning following diagnosis. 
This study had some limitations. First, our study was cross-sectional which fails to represent the dynamic nature of the cancer trajectory and the adaption process for patients and siblings. Second, the study comprised a relatively small sample which may have resulted in a lack of statistical power in testing some of the relationships between the variables.

Furthermore, as previously mentioned, the authors acknowledge the important conceptual distinction of cancer patients and their sibling's experiences of cancer diagnosis and treatment, and therefore the limitations associated with merging the samples for analyses. In this regard, the regression model outlined would also need to be tested in further studies. Third, it would have been useful to include multi-informant data in assessing family functioning (specifically self-report data from patients and siblings) and perceived social support (specifically self-report data from parents). Indeed, previous studies examining family functioning in childhood cancer and siblings have demonstrated that PTSS was predicted by self-report but not parent-reported family functioning. ${ }^{57}$, 58 This may provide some explanation for the lack of association between family functioning and PTSS in the current study. The study would have also benefitted from the inclusion of a measure of anxiety. This was omitted in the current study in an attempt to limit the length of the questionnaire battery, particularly for those young people who were experiencing fatigue in the context of cancer treatment.

Alongside addressing the above limitations, future research with this population may also benefit from examining other features implicated within cognitive models of PTSD, including rumination, safety behaviors and memory quality. Indeed, it is possible that cancer patients who do not adequately encode memories in the initial phase after their diagnosis may be more prone to subsequent PTSS because they cannot integrate and process their experiences optimally. This may also be an important factor for siblings who might also experience difficulties encoding illness related information. ${ }^{59}$ 
Finally, given the growing literature on posttraumatic growth (PTG; a process

whereby those who have experienced a traumatic event apply positive interpretations to and find meaning in the traumatic event) in children with cancer ${ }^{60}$ and their siblings ${ }^{61}$, it may be of interest to unpick factors that lead to PTG as opposed to posttraumatic stress to shed light on potential mediating variables, which could supplement current interventions. 


\section{References}

1. Ehlers A, Clark, DM. A cognitive model of posttraumatic stress disorder. Behaviour Research and Therapy. 2000; 38(4): 319-345.

2. Ehlers A, Steil, R. Maintenance of intrusive memories in posttraumatic stress disorder: a cognitive approach. Behavioural and Cognitive Psychotherapy. 1995; 23(3): 217-249.

3. American Psychiatric Association. Diagnostic and statistical manual of mental disorders. 5th ed. Arlington, VA: American Psychiatric Publishing; 2013.

4. Armour C, Müllerová J, Elhai JD. A systematic review of PTSD’s latent structure in the Diagnostic and Statistical Manual of Mental Disorders: DSM-IV to DSM-5. Clinical Psychology Review. 2016; 44: 60-74.

5. Breslau N, Lucia VC, Davis GC. Partial PTSD versus full PTSD: an empirical examination of associated impairment. Psychological Medicine. 2004; 34(7): 1205-1214.

6. Burnet KL, Brewin CR, Watson M. Intrusive cognitions and anxiety in cancer patients, Psychooncology. 2007; 16(5):132-133.

7. Kazak AE. Posttraumatic stress disorder (PTSD) and posttraumatic stress symptoms (PTSS) in families of adolescent childhood cancer survivors. Journal of Pediatric Psychology. 2004; 29(3): 211-219.

8. Butler RW, Rizzi LP, Handwerger BA. The assessment of posttraumatic stress disorder in pediatric cancer patients and survivors. Journal of Pediatric Psychology. 1996; 21(4): 499-504.

9. Erickson SJ, Steiner H. Trauma and personality correlates in long-term pediatric cancer survivors. Child Psychiatry and Human Development. 2001; 31(3):195-213.

10. Kazak AE, Boeving CA, Alderfer MA, Hwang WT, Reilly A. Posttraumatic stress symptoms during treatment in parents of children with cancer. Journal of Clinical 
Oncology. 2005; 23(30): 7405-7410.

11. Varela VS, Ng A, Mauch P, Recklitis CJ. Posttraumatic stress disorder (PTSD) in survivors of Hodgkin's lymphoma: prevalence of PTSD and partial PTSD compared with sibling controls. Psycho-oncology. 2013; 22(2): 434-40.

12. Sahler OJZ, Fairclough DI, Phipps S, et al. Using problem solving skills training to reduce negative affectivity in mothers of children with newly diagnosed cancer: report of a multisite randomized trial. Journal of Consulting and Clinical Psychology. 2005; 73(2): $272-283$.

13. Sahler OJZ, Dolgin MJ, Phipps S, et al. Specificity of problem-solving skills training in mothers of children newly diagnosed with cancer: results of a multisite randomized clinical trial. Journal of Clinical Oncology. 2013; 31(10): 1329-1335.

14. Shemesh E, Lurie S, Stuber ML, et al. A pilot study of posttraumatic stress and nonadherence in pediatric liver transplant recipients. Pediatrics. 2000; 105(2): 29-36.

15. Saxe G, Vanderbilt D, Zuckerman B. Traumatic stress in injured and ill children. PTSD Research Quarterly. 2003;14(2): 1-7.

16. Taïeb O, Moro MR, Baubet,T, Revah-Lévy A, Flament MF. Posttraumatic stress symptoms after childhood cancer. European Child \& Adolescent Psychiatry. 2003; 12(6): 255-264.

17. Tedstone JE, Tarrier N. Posttraumatic stress disorder following medical illness and treatment. Psychological Review. 2003; 23(3): 409-448.

18. Gurevich M, Devins GM, Rodin GM. Stress response syndromes and cancer: conceptual and assessment issues. Psychosomatics. 2002; 43(4): 259-281.

19. American Psychiatric Association. Diagnostic and statistical manual of mental disorders. $2^{\text {nd }}$ ed. Washington, DC: American Psychiatric Publishing; 1994.

20. Bruce M. A systematic and conceptual review of posttraumatic stress in childhood cancer 
survivors and their parents. Clinical Psychology Review. 2006; 26(3): 233-256.

21. National Institute for Health and Clinical Excellence. Improving outcomes in children and young people with cancer: Manual Update. London: National Institute for Health and Clinical Excellence; 2005.

22. Alderfer MA, Long KA, Lown EA, et al. Psychosocial adjustment of siblings of children with cancer: a systematic review. Psycho-oncology. 2010;19(8): 789-805.

23. Alderfer MA, Labay LE, Kazak A. Brief report: Does posttraumatic stress apply to siblings of childhood cancer survivors? Journal of Pediatric Psychology. 2003; 8(4): 281286.

24. Stuber ML, Meeske KA, Leisenring W, et al. Defining medical posttraumatic stress among young adult survivors in the Childhood Cancer Survivor Study. General Hospital Psychiatry. 2011; 33(4): 347-353.

25. Kangas M, Henry JL, Bryant RA. Predictors of posttraumatic stress disorder following cancer. Health psychology. 2005; 24(6): 579-85.

26. Brewin CR, Holmes EA. Psychological theories of posttraumatic stress disorder. Clinical Psychology Review. 2003; 23(3): 339-376.

27. Meiser-Stedman R. Towards a cognitive-behavioral model of PTSD in children and adolescents. Clinical Child and Family Psychology Review. 2002; 5(4): 217-232.

28. Smith P, Yule W, Perrin S, Tranah T, Dalgleish T, Clark DM. Cognitive-behavioral therapy for PTSD in children and adolescents: a preliminary randomized controlled trial. Journal of the American Academy of Child and Adolescent Psychiatry. 2007; 46(8): $1051-1061$.

29. Stallard P. A retrospective analysis to explore the applicability of the Ehlers And Clark (2000) cognitive model to explain PTSD in children. Behavioural and Cognitive Psychotherapy. 2003; 31(3): 337-345. 
30. Trickey D, Siddaway AP, Meiser-Stedman R, Serpell L, Field AP. A meta-analysis of risk factors for post-traumatic stress disorder in children and adolescents. Clinical Psychology Review. 2012; 32(2); 122-138.

31. Salmon K, Bryant RA. Posttraumatic stress disorder in children: The influence of developmental factors. Clinical Psychology Review. 2002; 22(2): 163-188.

32. Meiser-Stedman RA, Yule W, Dalgleish T, Smith P, Glucksman, E. The role of the family in child and adolescent posttraumatic stress following attendance at an emergency department. Journal of Pediatric Psychology. 2005; 31(4): 397-402.

33. Williamson V, Creswell C, Fearon P, Hiller RM, Walker J, Halligan SL. The role of parenting behaviors in childhood post-traumatic stress disorder: A meta-analytic review. Clinical Psychology Review. 2017; 53: 1-13.

34. Pine DS, Cohen JA. Trauma in children and adolescents: Risk and treatment of psychiatric sequelae. Biological psychiatry. 2002; 51(7): 519-531.

35. Pynoos RS, Steinberg AM, Piacentini JC. A developmental psychopathology model of childhood traumatic stress and intersection with anxiety disorders. Biological psychiatry. 1999; 46(11): 1542-1554.

36. Weiss DS, Marmar CR. The impact of event scale-revised. In: Wilson JP \& Keane TM. Accessing Psychological Trauma and PTSD. New York: The Guilford Press; 1997: 399411.

37. Best M, Streisand R, Catania L, Kazak AE. Parental distress during pediatric leukemia and posttraumatic stress symptoms (PTSS) after treatment ends. Journal of Pediatric Psychology. 2001; 26(5): 299-307.

38. Meiser-Stedman R, Dalgleish T, Glucksman E, et al. Maladaptive cognitive appraisals mediate the evolution of posttraumatic stress reactions: A 6-month follow-up of child and adolescent assault and motor vehicle accident survivors. Journal of Abnormal 
Psychology. 2009; 118(4): 778-787.

39. Foa EB, Ehlers A, Clark DM, Tolin DF, Orsillo SM. The Posttraumatic Cognitions Inventory (PTCI): development and validation. Psychological Assessment. 1999; 11(3): 303-314.

40. Berntsen D, Rubin DC. The centrality of event scale: a measure of integrating a trauma into one's identity and its relation to post-traumatic stress disorder symptoms. Behaviour Research and Therapy. 2006; 44(2): 219-231.

41. Miller IW, Epstein NB, Bishop DS, Keitner GI. The McMaster family assessment device: Reliability and validity. Journal of Marital and Family Therapy. 1985; 11(4): 345-356.

42. Lewandowski AS, Palermo TM, Stinson J, Handley S, Chambers CT. Systematic review of family functioning in families of children and adolescents with chronic pain. The Journal of Pain. 2010; 11(11): 1027-1038.

43. Zimet GD, Dahlem NW, Zimet SG, Farley GK. The multidimensional scale of perceived social support. Journal of Personality Assessment. 1988; 52(1): 30-41.

44. Tabachnick BG, Fidell LS. Computer-assisted research design and analysis. Boston: Allyn and Bacon; 2001.

45. Pallant J. SPSS survival manual. McGraw-Hill Education (UK); 2013.

46. Wright S.P. Adjusted p-values for simultaneous inference. Biometrics. 1992; 1005-1013.

47. Creamer M, Bell R, Failla S. Psychometric properties of the impact of event scale revised. Behaviour Research and Therapy. 2003; 41(12): 1489-1496.

48. Currier JM, Jobe-Shields LE, Phipps S. Stressful life events and posttraumatic stress symptoms in children with cancer. Journal of Traumatic Stress. 2009; 22(1): 28-35.

49. Wilkins KL, Woodgate RL. A review of qualitative research on the childhood cancer experience from the perspective of siblings: A need to give them a voice. Journal of Pediatric Oncology Nursing. 2005; 22(6): 305-319. 
50. Hardy KK, Willard VM, Watral MA, Bonner MJ. Perceived social competency in children with brain tumors: comparison between children on and off therapy. Journal of Pediatric Oncology Nursing. 2010; 27(3): 156-163.

51. von Essen L, Enskar K, Kreuger A, Larsson B, Sjoden PO. Self-esteem, depression and anxiety among Swedish children and adolescents on and off cancer treatment. Acta Paediatrica. 2000; 89(2): 229-236.

52. Alderfer MA, Hodges JA. Supporting siblings of children with cancer: A need for familyschool partnerships. School Mental Health. 2010; 2(2): 72-81.

53. Rodriguez EM, Dunn MJ, Zuckerman T, Vannatta K, Gerhardt CA, Compas BE. Cancerrelated sources of stress for children with cancer and their parents. Journal of Pediatric Psychology. 2012; 37(2): 1-13.

54. Green BL, Krupnick JL, Rowland JH, Epstein SA, Stockton P, Spertus I, Stern N. Trauma history as a predictor of psychologic symptoms in women with breast cancer. Journal of Clinical Oncology. 2000; 18(5): 1084-1093.

55. Kangas M, Henry JL, Bryant RA. Posttraumatic stress disorder following cancer: A conceptual and empirical review. Clinical Psychology Review. 2002; 22(4): 499-524.

56. Kazak AE, Simms S, Alderfer M, et al. Feasibility and preliminary outcomes from a pilot study of brief psychological intervention for families of children newly diagnosed with cancer. Journal of Pediatric Psychology. 2005; 30(8): 644-655.

57. Long KA, Marsland AL, Alderfer MA. Cumulative family risk predicts sibling adjustment to childhood cancer. Cancer. 2013; 119(13): 2503-2510.

58. Pelcovitz D, Libov BG, Mandel F, Kaplan S, Weinblatt M, Septimus A. Posttraumatic stress disorder and family functioning in adolescent cancer. Journal of Traumatic Stress. 1998; 11(2): 205-221.

59. Houtzager BA, Grootenhuis MA, Last BF. Supportive groups for siblings of pediatric 
oncology patients: impact on anxiety. Psycho-oncology. 2001; 10(4): 315-24.

60. Barakat LP, Alderfer MA, Kazak AE. Posttraumatic growth in adolescent survivors of cancer and their mothers and fathers. Journal of Pediatric Psychology. 2006; 31(4): 413319.

61. D’Urso A, Mastroyannopoulou K, Kirby A. Experiences of posttraumatic growth in siblings of children with cancer. Clinical child psychology and psychiatry. 2017; 22(2): 301-17. 
Patients meeting eligibility criteria and approached by staff $(n=52)$

Families contacting researcher independently via poster $(n=6)$ Families consenting to be contacted $(n=31 ; 60 \%)$

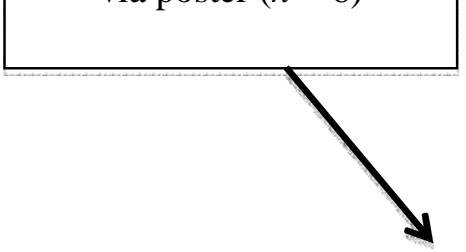

Families providing informed consent and participating in research Total families ( $n=37)$; patient-sibling pairs $(n=22)$; Independent patients $(n=12)$; Independent siblings $(n=4)$

Figure 1: Recruitment Flow Chart 
Table 1. Demographic and Medical Information of Sample $(N=60)$

\begin{tabular}{|c|c|c|}
\hline Characteristic & $\begin{array}{l}\text { Patients } \\
(n=34)\end{array}$ & $\begin{array}{l}\text { Siblings } \\
(n=26) \\
* \text { Corresponding } \\
\text { data }\end{array}$ \\
\hline Age [mean (SD)] & $12.24(2.81)$ & $12.58(2.94)$ \\
\hline \multicolumn{3}{|l|}{ Gender $[n(\%)]$} \\
\hline Male & $13(38)$ & $14(54)$ \\
\hline Female & $21(62)$ & $12(46)$ \\
\hline \multicolumn{3}{|l|}{ Diagnostic category $[n(\%)]$} \\
\hline ALL $^{\mathrm{a}}$ & $18(53)$ & $16(61)$ \\
\hline Other Leukemia & $1(3)$ & $2(8)$ \\
\hline $\mathrm{HD} / \mathrm{NHL}^{\mathrm{b}}$ & $4(12)$ & $1(4)$ \\
\hline Solid tumor & $6(17)$ & $3(12)$ \\
\hline Brain tumor & $5(15)$ & $4(15)$ \\
\hline \multicolumn{3}{|l|}{ Treatment Received $[n(\%)]$} \\
\hline Chemotherapy & $20(59)$ & $13(50)$ \\
\hline Radiotherapy \& Surgery & $2(6)$ & $2(8)$ \\
\hline Chemotherapy \& Surgery & $4(12)$ & $2(8)$ \\
\hline Chemotherapy \& Radiotherapy & $2(6)$ & $4(15)$ \\
\hline Transplant $^{\mathrm{c}}$ & $6(17)$ & $5(19)$ \\
\hline \multicolumn{3}{|l|}{ Treatment Stage $[n(\%)]$} \\
\hline Ongoing & $11(32)$ & $9(35)$ \\
\hline Completed & $23(68)$ & $17(65)$ \\
\hline \multicolumn{3}{|l|}{ Time Since Diagnosis [n (\%)] } \\
\hline$\geq 1-9$ months & $5(15)$ & $3(12)$ \\
\hline$\overline{9}-24$ months & $11(32)$ & $6(23)$ \\
\hline 24 months to 5 years & $18(53)$ & $17(65)$ \\
\hline \multicolumn{3}{|l|}{ Duration of Treatment $[n(\%)]$} \\
\hline$\geq 1-6$ months & $7(21)$ & $4(15)$ \\
\hline $6-12$ months & $4(12)$ & $5(19)$ \\
\hline $12-24$ months & $8(23)$ & $3(12)$ \\
\hline $24-40$ months & $15(44)$ & $14(54)$ \\
\hline
\end{tabular}

*Note: Given previous research suggesting poorer psychological adjustment in parents according to specific cancer diagnoses and treatments (Fuemmeler et al., 2001; Bruce, 2006), descriptive data pertaining to the cancer diagnosis and treatment 'experienced' by siblings is included; $a=$ Acute lymphocytic leukemia; $b=$ Hodgkin's disease/non-Hodgkin's lymphoma; $c=$ Included organ transplant and bone marrow transplant in conjunction with chemotherapy, radiotherapy or both. 
Table 2. Primary Stressors Reported on IES-R

\begin{tabular}{lcc}
\hline Primary Stressor $[\boldsymbol{n}(\boldsymbol{\%})]$ & Patients $(\boldsymbol{n}=\mathbf{3 4})$ & Siblings $(\boldsymbol{n}=\mathbf{2 6})$ \\
\hline Diagnosis & $28(82)$ & $20(77)$ \\
Admission to Intensive Care & $3(9)$ & $4(15)$ \\
Seeing sibling in an ambulance & - & $2(8)$ \\
Surgery & $3(9)$ & - \\
\hline
\end{tabular}

12

13

14

15

16 
Table 3. Correlations between PTSS and Demographic, Treatment-Related, Cognitive and Environmental Variables

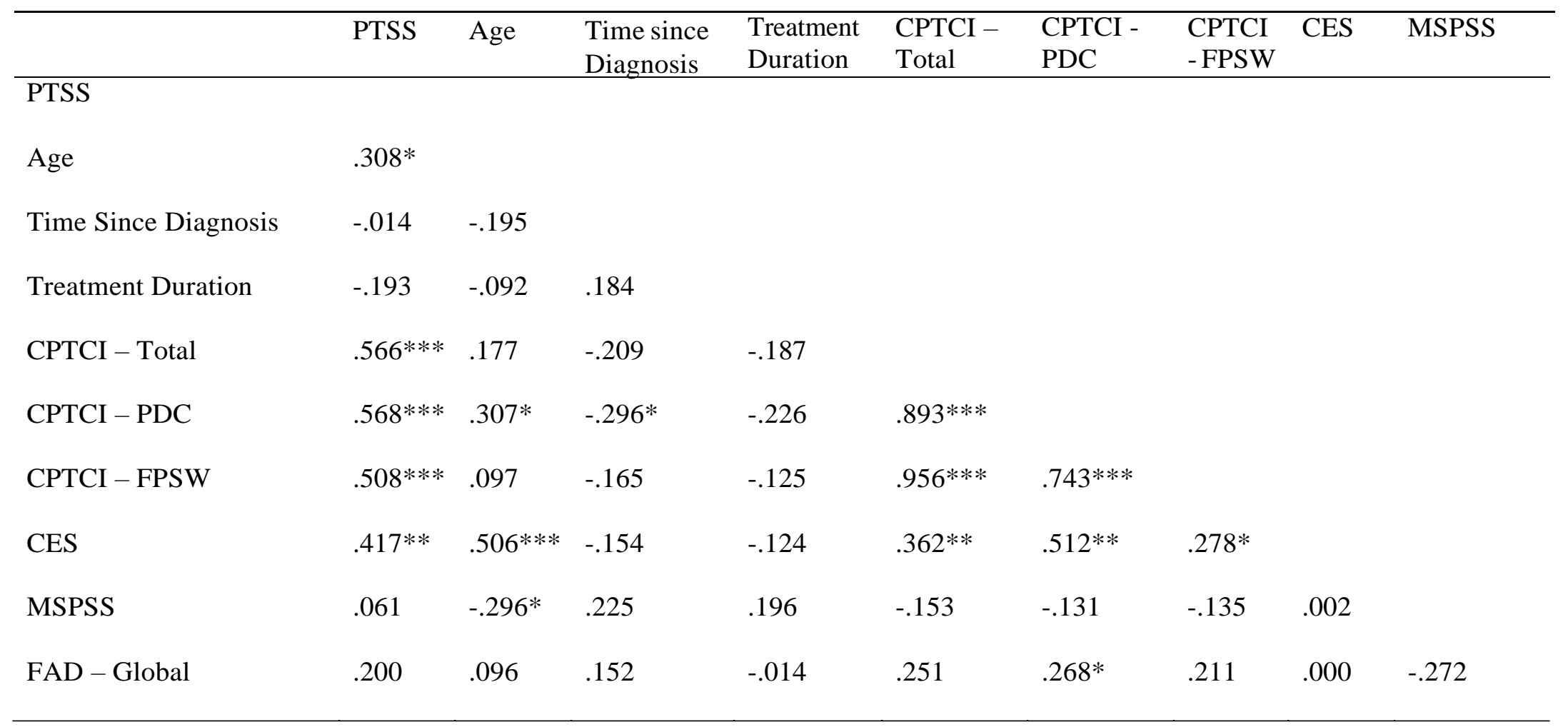

Note: PTSS = Posttraumatic Stress Symptoms; CPTCI-PDC = Post-Traumatic Cognitions Inventory-Child Version, Permanent and Disturbing Change subscale; CPTCI-FPSW = CPTCI, Fragile Person in a Scary World subscale, CES=Centrality of Events Scale; MSPSS = Multidimensional Scale of Perceived Social Support; FAD Global = Family Assessment Device Global Scale; These analyses reflect Spearman's rho correlation co-efficients; $* p<.05 . * * p . \leq .01 . * * * p \leq 001$. 
Table 4. Regression modelling of post-traumatic stress symptoms in all youth.

\begin{tabular}{|c|c|c|c|c|c|}
\hline & Model $\mathrm{R}^{2}$ & $F$ test & $\Delta \mathrm{R}^{2}$ & $F$ test & $\begin{array}{c}\text { Beta } \\
\text { (step two) }\end{array}$ \\
\hline \multicolumn{6}{|l|}{ MODEL ONE } \\
\hline $\begin{array}{l}\text { Step one } \\
\text { Age }\end{array}$ & .15 & $\mathrm{~F}_{1,56}=9.65^{* *}$ & - & - & .19 \\
\hline $\begin{array}{l}\text { Step two } \\
\text { CPTCI } \\
\text { CES }\end{array}$ & .37 & $\mathrm{~F}_{3,54}=10.46^{* *}$ & .22 & $\mathrm{~F}_{2,54}=9.41^{* * *}$ & $\begin{array}{l}.38^{* *} \\
.22\end{array}$ \\
\hline \multicolumn{6}{|l|}{ MODEL TWO } \\
\hline Step one & .16 & $\mathrm{~F}_{2,55}=5.10^{* *}$ & - & - & \\
\hline Age & & & & & .17 \\
\hline Child status & & & & & .13 \\
\hline $\begin{array}{l}\text { Step two } \\
\text { CPTCI } \\
\text { CES }\end{array}$ & .38 & $\mathrm{~F}_{4,53}=8.25^{* * *}$ & .23 & $\mathrm{~F}_{2,53}=9.78^{* * *}$ & $\begin{array}{l}.37^{* *} \\
.25\end{array}$ \\
\hline
\end{tabular}

Note $:$ CPTCI $=$ Child Post-Traumatic Cognitions Inventory; CES $=$ Centrality of Events Scale

$\mathrm{p}<.01,{ }^{* * *}=\mathrm{p}<.001$ 